\title{
Genetic and Structural Evidence for the Presence of Propanediol Oxidoreductase Isoenzymes in Escherichia coli
}

\author{
By JOAQUIM ROS AND JUAN AGUILAR* \\ Department of Biochemistry, School of Pharmacy, University of Barcelona (Pedralbes), \\ Barcelona-28, Spain
}

(Received 28 April 1983; revised 22 August 1983)

\begin{abstract}
The synthesis of propanediol oxidoreductase, an enzyme permitting the anaerobic metabolism of fucose and rhamnose, has been described as being controlled by the prd locus closely linked to the fuc locus in wild-type cells of Escherichia coli. However, strain AA-787, deleted in the fuc and prd loci, grew anaerobically on rhamnose, displaying propanediol oxidoreductase activity. From the deleted strain we derived a constitutive producer of propanediol oxidoreductase able to grow on 1,2-propanediol by oxidizing the diol to lactaldehyde which was further metabolized to lactate. Transduction experiments showed that this ability to use propanediol was closely linked to the rha locus. Peptide mapping of fucose- and rhamnose-induced propanediol oxidoreductase of wild-type cells established structural differences between the two enzymes, indicating two structural genes, one for each sugar metabolizing system.
\end{abstract}

\section{INTRODUCTION}

Propanediol oxidoreductase (EC 1.1.1.77) is an enzyme of the fucose and rhamnose fermentation pathways in Escherichia coli (Cocks et al., 1974; Boronat \& Aguilar, 1979). Both pathways converge after the cleavage of fuculose-1-phosphate and rhamnulose-1-phosphate, yielding the same two products, dihydroxyacetone phosphate and L-lactaldehyde. The oxidoreductase converts the $L$-lactaldehyde formed into $L-1,2$-propanediol which is excreted into the medium as a fermentation product (Cocks et al., 1974).

As expected, propanediol oxidoreductase is induced by anaerobic growth of wild-type $E$. coli on fucose (Cocks et al., 1974) and rhamnose (Boronat \& Aguilar, 1979). However, mutants of the wild-type strain may be isolated in which the production of propanediol oxidoreductase is constitutive. In these, the enzyme acts by converting L-1,2-propanediol to lactaldehyde, thus permitting growth of these cells on L-1,2-propanediol (Sridhara et al., 1969). Acquisition of this catabolic pathway depends on a genetic alteration of the dissimilatory system for L-fucose, on which most of the mutants are no longer able to grow (Hacking \& Lin, 1976).

This constitutive production of propanediol oxidoreductase, phenotypically expressed as the ability to grow on L-1,2-propanediol, has been located by transduction very close to the relA gene (Russell, 1973) and has been shown to co-transduce with,100\% frequency with the fuc locus (Hacking et al., 1978).

The induction of propanediol oxidoreductase by anaerobic growth on rhamnose (Boronat \& Aguilar, 1979), the observation of differences between the fucose and rhamnose enzyme induction (Boronat \& Aguilar, $1981 b$ ) and the description of two independent sets of enzymes for the aerobic metabolism of fucose (Green \& Cohen, 1956; Ghalambor \& Heath, 1962; Heath \& Ghalambor, 1962) and rhamnose (Takagi \& Sawada 1964a,b; Chiu \& Feingold, 1969) which are coded by genes located $27 \mathrm{~min}$ apart in the $E$. coli chromosome, led us to search for a gene for propanediol oxidoreductase linked to the rha operon. 


\section{METHODS}

Bacieria and phage. The wild-type strain was a derivative of E. coli K12, strain E-15 (Bachmann, 1972) and here referred to as strain 1 . Strain 3 was derived from strain 1 as a propanediol oxidoreductase mutant after ethyl methanesulphonate mutagenesis (Sridhara et al., 1969). These two strains were from the laboratory of E. C. C. Lin, Department of Microbiology and Molecular Genetics, Harvard Medical School, Boston, MA 02115, USA. A mutant of $E$. coli K12, strain AA-787, containing a large deletion encompassing argA, fuc and relA (Atherly, 1979) was kindly provided by A. G. Atherly, Department of Genetics, lowa State University, Ames, IA 50011, USA. This strain was also arg $H$, thr, leu, his, thi and thyA. Strain DF-903 containing a deletion from rha to pfkA (Daldal \& Fraenkel, 1981) was given to us by D. G. Fraenkel, Department of Microbiology and Molecular Genetics, Harvard Medical School, Boston, MA 02115, USA. Transduction with phage P1 was carried out by the method of Miller (1972).

Chemicals. DL-1,2-propanediol was obtained from Merck, Darmstadt, F.R.G. and purified by distillation. LLactaldehyde was prepared in our laboratory by the procedure of Boronat \& Aguilar (1979). L-Fucose, L-rhamnose and NADH were obtained from Sigma, and casein acid hydrolysate was from Difco. The enzyme $\alpha$-chymotrypsin from beef pancreas was obtained from Worthington, Freehold, NJ, USA. Gel electrophoresis materials were from Bio-Rad. All other materials were reagent grade and obtained from commercial sources.

Growth of cells. Cells were grown as indicated by Boronat \& Aguilar (1979). Strain AA-787 was grown in the same media supplemented with amino acids at 10 to $50 \mu \mathrm{g} \mathrm{m} \mathrm{m}^{-1}$ depending upon their ratio in proteins (or by adding $0.2 \%$ casein acid hydrolysate), thiamin $\left(1 \mu \mathrm{g} \mathrm{ml}{ }^{-1}\right)$ and thymidine $\left(4 \mu \mathrm{g} \mathrm{ml}^{-1}\right)$.

Preparation of cell extracts and enzyme assay. Cell extracts were prepared and enzymes assayed as described previously (Boronat Aguilar, 1981 b). One unit of enzyme activity was defined as the amount of enzyme that converted $1 \mu \mathrm{mol}$ substrate $\min ^{-1}$.

Concentration of protein in cell extracts was determined by the Lowry method, using bovine serum albumin as standard.

Immonological techniques. Antisera against propanediol oxidoreductase were obtained as described previously (Boronat \& Aguilar, 1981 b). Double immunodiffusion and quantitative immunoelectrophoresis were performed as described by Ouchterlony (1953) and Laurell (1966), respectively.

Peptide mapping. For peptide mapping, propanediol oxidoreductase from strain 1 grown on fucose or rhamnose was purified as described previously (Boronat \& Aguilar, 1979). In order to eliminate some minor contaminants, the enzyme preparations were further purified by electrophoresis according to Laemmli (1970). After a brief staining, the band corresponding to propanediol oxidoreductase was sliced from the gel and electrophoretically eluted into a dialysis bag using $0.1 \%(\mathrm{w} / \mathrm{v})$ SDS in $2.5 \mathrm{mu}$-Tris/glycine (pH 8.3) as buffer. The protein content of the bag was precipitated with nine volumes of cold acetone, resuspended in $100 \mathrm{~mm}$-ammonium bicarbonate and dialysed overnight against the same buffer. The samples were lyophylized in $1 \mathrm{ml}$ conical glass vials and taken up in $0.05 \%(w / v)$ SDS, $100 \mathrm{~mm}$-ammonium bicarbonate (pH 8.0) at a protein concentration of $1 \mathrm{mg} \mathrm{ml}^{-1}$.

The protein was then digested with the chymotrypsin under the conditions indicated below. A sample of $1 \mathrm{nmol}$ protein digest was spotted on a cellulose thin-layer plate that had previously been washed overnight by ascending chromatography in butanol/pyridine/acetic acid/water (50:33:1:40, by vol.). Electrophoresis in the first dimension was carried out in a Varsol cooled apparatus (Whittaker \& Moss, 1981), using pyridine/acetic acid/water $(1: 10: 89$, by vol.) as buffer and applying $900 \mathrm{~V}$ for $30 \mathrm{~min}$. After drying, the plates were kept at room temperature for $10 \mathrm{~min}$ and then chromatographed at right angles to the first dimension in the butanol/pyridine/ acetic acid/water solvent. The dried, cooled plates were sprayed with $0 \cdot 1 \%(w / v)$ fluorescamine in acetone, and the peptides visualized under long-wavelength UV light.

\section{RESULTS}

Growth properties of strain AA-787

As the fuc and prd loci are deleted in strain AA-787 it did not grow on fucose either aerobically or anaerobically. This strain, as expected, grew aerobically on rhamnose with a doubling time of $110 \mathrm{~min}$ and, in spite of the lack of the prd locus, also grew anaerobically on rhamnose with a doubling time of $260 \mathrm{~min}$. The yields of aerobic and anaerobic cultures of strain AA-787 were similar to those obtained in the same conditions with wild-type $E$. coli.

\section{Propanediol oxidoreductase in strain AA-787}

Strain AA-787 displayed a very low propanediol oxidoreductase activity when grown aerobically on casein acid hydrolysate or rhamnose. However, this activity was induced five- to sixfold when it was grown on rhamnose anaerobically (Table 1). 
Table 1. Activity of propanediol oxidoreductase in crude extracts of strains AA-787 and JA-101 grown under different conditions

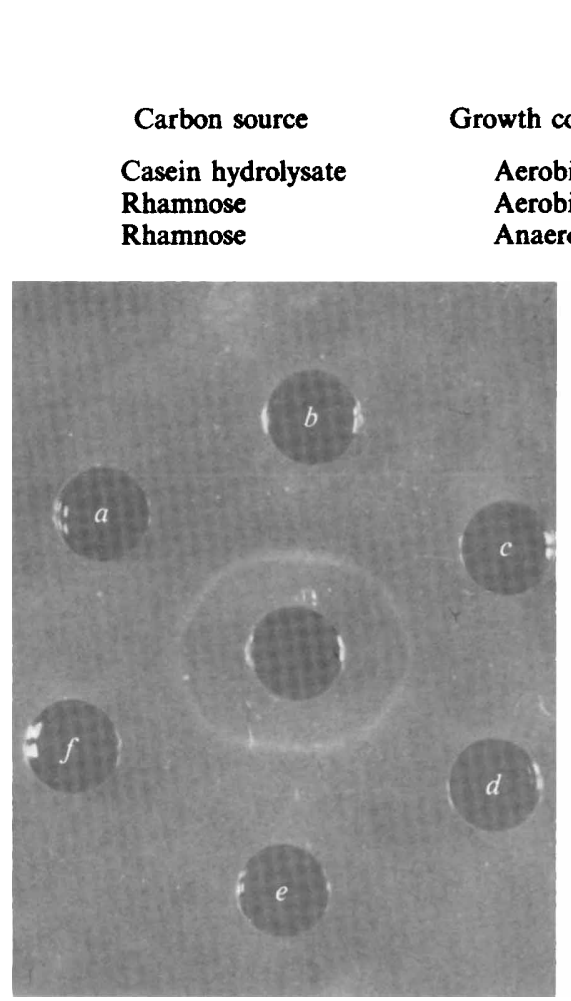

Fig. 1.

$\overbrace{\text { AA-787 }}^{\text {[units (mg protein) }^{-1} \text { ] }} \begin{array}{cc}\text { Enzyme activity } \\ 0.10 & \text { JA-101 } \\ 0.08 & 0.48 \\ 0.50 & 0.44 \\ & 0.61\end{array}$

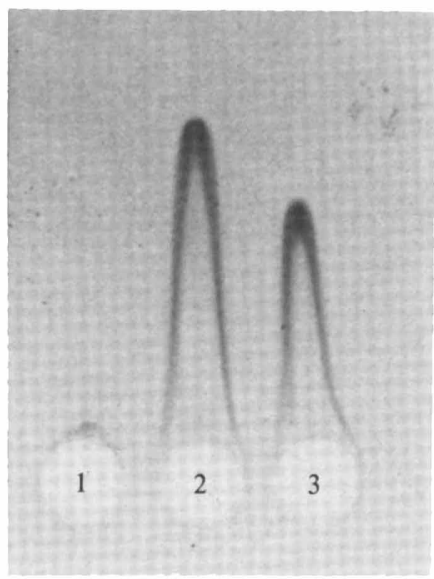

Fig. 2.

Fig. 1. Immunological identity between propanediol oxidoreductase of strain 1, strain 3 and strain AA787. Wells in the Ouchterlony immunodiffusion plates contained $25 \mu \mathrm{l}$ cell extracts of: (a) strain 1 grown anaerobically on fucose $(30 \mu \mathrm{g}$ protein), $(b)$ and $(e)$ strain 3 grown aerobically on casein hydrolysate (6 $\mu \mathrm{g}$ protein), (c) strain 1 grown anaerobically on rhamnose ( $30 \mu \mathrm{g}$ protein) and $(d)$ and $(f)$

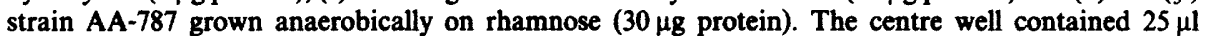
antiserum.

Fig. 2. Immunoelectrophoretic quantification of propanediol oxidoreductase. The Laurell rockets were obtained by applying to a gel containing $1.5 \%$ antiserum, $25 \mu \mathrm{g}$ protein in $10 \mu \mathrm{l}$ cell extract of : (1) strain AA-787 grown aerobically on casein hydrolysate, (2) strain AA-787 grown anaerobically on rhamnose and (3) strain JA-101 grown aerobically on casein hydrolysate.

Propanediol oxidoreductase induced in strain AA-787 appeared identical to the wild-type enzyme by double immunodiffusion against enzyme antibodies (Fig. 1). This permitted us to quantify the amount of enzyme by Laurell immunoelectrophoresis of crude extracts. Under our conditions, the length of the Laurell rocket was proportional to the amount of enzyme over a wide range of concentrations. The enzymic protein was almost non-existent in strain AA-787 grown under non-inducing conditions, as shown by the absence of a rocket when such extracts were analysed. In agreement with the presence of enzymic activity in extracts of cells grown under inducing conditions, the rocket was apparent in the immunoelectrophoresis of such extracts (Fig. 2).

\section{A propanediol oxidoreductase constitutive mutant of strain $A A-787$}

A constitutive producer of propanediol oxidoreductase was derived from strain AA-787 by isolating mutants able to grow on 1,2-propanediol (Sridhara et al., 1969). Selection was performed on DL-1,2-propanediol plates inoculated with an ethyl methanesulphonate 


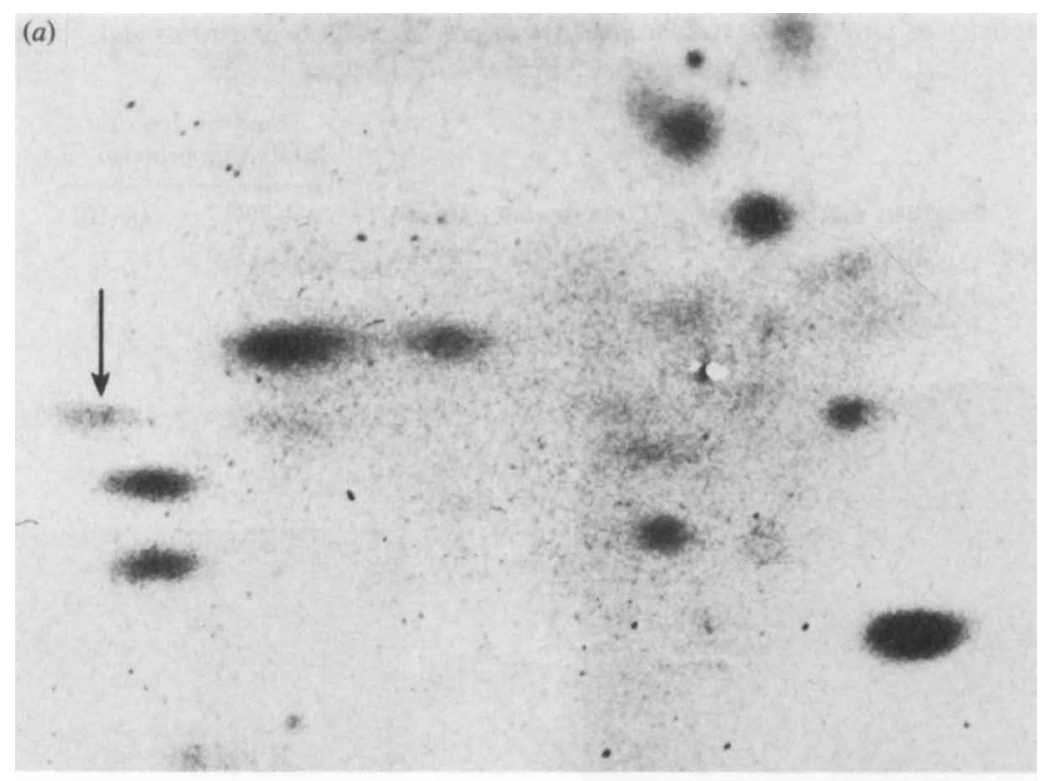

(b)

Fig. 3. Peptide mapping of purified propanediol oxidoreductase from strain 1 grown anaerobically on (a) fucose or (b) rhamnose. Arrows indicate spots present on one map but not on the other.

mutagenized population as described by Lin et al. (1962). A clone (strain JA-101) was chosen for its good growth on these plates and was subsequently restreaked four times on the same medium. This strain grew aerobically on propanediol as a sole source of carbon and energy with a doubling time of $95 \mathrm{~min}$ and also grew on rhamnose aerobically and anaerobically.

Extracts of strain JA-101 exhibited propanediol oxidoreductase activity when the cells were grown under inducing as well as under non-inducing conditions (Table 1). Furthermore, this enzymic activity could be correlated with the production of propanediol oxidoreductase protein as measured by the length of the corresponding Laurell rocket (Fig. 2).

The ability of strain JA-101 to grow on propanediol co-transduced with the rha locus was shown by an experiment in which strain DF-903 carrying a deletion in the rha locus was transduced 
with P1 phage grown on strain JA-101. An estimated $80 \%$ of the transductants that recovered the ability to grow on rhamnose also acquired the ability to grow on propanediol. The frequency of mutation to the ability to grow on propanediol as a result of the constitutive production of the propanediol oxidoreductase linked to the fuc locus was far below the number of transductants plated.

\section{Structural differences between fucose and rhamnose induced propanediol oxidoreductase}

Propanediol oxidoreductase of strain 1 grown anaerobically on fucose or rhamnose was purified as indicated in Methods. The purified proteins were digested with $\alpha$-chymotrypsin in a propanediol oxidoreductase to chymotrypsin ratio of $100: 1(\mathrm{w} / \mathrm{w})$ by incubating the mixture at $37^{\circ} \mathrm{C}$ for $10 \mathrm{~h}$ with gentle stirring. Before being electrophoresed and chromatographed as indicated in Methods, the samples were concentrated 10-fold using a stream of dry nitrogen at $40{ }^{\circ} \mathrm{C}$. The peptide maps obtained are shown in Fig. 3. The arrows indicate the main differences between the spot patterns obtained with the enzymes induced by fucose and rhamnose.

\section{DISCUSSION}

As discussed by Boronat \& Aguilar (1981 $b$ ) there are two possibilities for the regulation of propanediol oxidoreductase during fucose and rhamnose fermentation. One is that both systems use the same propanediol oxidoreductase linked to the fuc locus (Bachman \& Low, 1980), although its expression is regulated in a different way; the other is that each system has its own propanediol oxidoreductase (coded by highly similar genes since their products cannot be easily distinguished).

If both sugars were fermented by the action of a single propanediol oxidoreductase specified by a gene linked to fuc, the lack of this enzyme produced by the deletion of the prd and fucloci would necessarily result in the inability of the deletion mutants to ferment either fucose or rhamnose. Neither genetic nor biochemical complementation would be possible in such mutants. Our results with strain AA-787 indicate that this is not the case; there is another means of obtaining propanediol oxidoreductase expression in these cells. The possible absence of propanediol oxidoreductase activity as a consequence of inhibition of enzyme activity by a biochemical regulatory mechanism was eliminated by the immunological quantification of the enzyme protein.

The results obtained with strain AA-787 and its derivative strain JA-101 confirm the presence of a regulatory gene, besides the one linked to fuc, acting on the expression of the oxidoreductase. The close linkage of this gene for oxidoreductase expression to rha was ascertained not only by the rhamnose induction of the activity but also by the co-transduction of constitutive propanediol oxidoreductase synthesis in strain JA-101 with the rha locus.

So far, no functional or structural difference between the fucose- and rhamnose-induced propanediol oxidoreductase has been described, but there are several reports of enzymes, indistinguishable by their functional parameters, that actually show differences in peptide mapping or amino acid sequencing (Koo \& Adams, 1974; Kikuchi \& Gorini, 1975; Boronat \& Aguilar, 1981a). Here we show structural differences as indicated by the different patterns of spots obtained in the two-dimensional peptide mapping of the purified enzymes induced by fucose or rhamnose. This is in accord with the existence of a regulatory system for propanediol oxidoreductase expression different from the one specified by a gene linked to fuc. It would also be compatible with the presence of two different structural genes of undetermined location in addition to the regulatory genes described - one close to the fuc locus, the other close to the rha locus.

The two similar sets of genes for the parallel metabolism of fucose and rhamnose lie $27 \mathrm{~min}$ apart on the map of the $E$. coli chromosome. According to the hypothesis of Riley \& Anilionis (1978) concerning the mechanism of evolution of the bacterial genome, these two sets of genes might be derived by duplication of the entire chromosome. Although the propanediol oxidoreductase genetic system is not included in the sets of genes described, the existence of a similar duplication for the oxidoreductase would not be surprising (Zipkas \& Riley, 1975). One of the 
two propanediol oxidoreductase genes may be lost or silent, although our results indicate that this is not the case.

This work was supported by 'Comisión Asesora de Investigación Científica y Técnica', Spain. We thank Laboratorios Ferrer Internacional for co-operation in the preparation of the enzyme antibody.

\section{REFBRENCES}

ATHERLY, A. G. (1979). Escherichia coli mutant containing a large deletion from relA to argA. Jownal of Bacteriology 138, 530-534.

Bachun, B. J. (1972). Pedigrees of some mutant strains of Escherichia coli $\mathrm{K}-12$. Bacteriological Reviews 36, 525-557.

BACHMANN, B. J. \& Low, K. B. (1980). Linkage map of Escherichia cali K-12, edition 6. Microbiological Reviews 44, 1-56.

Boronat, A. \& Aguilar, J. (1979). Rhamnoseinduced propanediol oxidoreductase in Escherichia coli: purification properties and comparison with the fucose-induced enzymes. Journal of Bacteriology $140,320-326$.

Boronat, A. \& AGUilar, J. (1981a). Experimental evolution of propanediol oxidoreductase in Escherichia coli: comparative analysis of the wild type and mutant enzymes. Biochimica et biophysica acta 672 , 98-107.

BORONAT, A. \& AgUILAR, J. (1981 b). Metabolism of Lfucose and L-rhamnose in Escherichia coli: differences in induction of propanediol oxidoreductase. Joumal of Bacteriology 147, 181-185.

ChIU, T. H. \& Fenngold, D. S. (1969). L-rhamnulose 1phosphate aldolase from Escherichia coli. Crystallization and properties. Biochemistry 8, 98-108.

Cocks, G. T., Aguilak, J. \& LiN, E. C. C. (1974). Evolution of L-1,2-propanediol catabolism in Escherichia coli by recruitment of enzymes for $L$-fucose and L-lactate metabolism. Journal of Bacteriology 118, 83-88.

Daldal, F. \& Fraenkel, D. G. (1981). Tnl0 insertions in the $p f k B$ region of Escherichia coli. Joumal of Bacteriology 147, 935-943.

Ghalambor, M. A. \& Heath, E. C. (1962). The metabolism of $L$-fucose. II. The enzymatic cleavage of L-fuculose-1-phosphate. Jowmal of Biological Chemistry 237, 2427-2433.

Green, M. \& Cohen, S. S. (1956). The enzymatic conversion of L-fucose to L-fuculose. Journal of Biological Chemistry 219, 557-568.

HACKING, A. J. \& LIN, E. C. C. (1976). Disruption of the fucose pathway as a consequence of genetic adaptation to propanediol as a carbon source in Escherichia coli. Journal of Bacteriology 126, 11661172.

Hacking, A. J., Aguilar, J. \& LIN, E. C. C. (1978). Evolution of propanediol utilization in Escherichia coli: mutant with improved substrate-scavenging power. Joumal of Bacteriology 136, 522-530.
Heath, E. C. \& Ghalambor, M. A. (1962). The metabolism of L-fucose. I. The purification and properties of L-fuculose kinase. Joumal of Biological Chemistry 237, 2423-2426.

KIxUCHI, A. \& GoRINI, L. (1975). Similarity of genes arg $F$ and argl. Nature, London 256, 621-624.

KoO, P. H. \& ADAws, E. (1974). $\alpha$-Ketoglutaric semialdehyde dehydrogenase of Pseudomonas. Properties of the separately induced isoenzymes. Journal of Biological Chemistry 249, 1704-1716.

LAEMMLI, U. K. (1970). Cleavage of structural proteins during the assembly of the head of bacteriophage T4. Nature, London 227, 680-685.

LAURELL, C. B. (1966). Quantitative estimation of proteins by electrophoresis in agarose gel containing antibodies. Analytical Biochemistry 15, 45-52.

LiN, E. C. C., Lerner, S. A. \& Jorgensen, S. E. (1962). A method for isolating constitutive mutants for carbohydrate-catabolizing enzymes. Biochimica et biophysica acia 60, 422-424.

Miller, J. H. (1972). Experiments in Molecular Genetics, Ist edn, pp. 230-234. Cold Spring Harbor, New York: Cold Spring Harbor Laboratory.

OUCHTBRLONY, O. (1953). Antigen-antibodies reactions in gels. IV. Types of reactions in coordinated systems of diffusion. Acta pathologica et microbiologica scandinavica 22, 231-240.

RILEY, M. \& ANILIONIS, A. (1978). Evolution of the bacterial genome. Annual Review of Microbiology 32, $519-560$.

Russell, R. R. B. (1973). Close linkage of prd and rel genes in Escherichia coli K-12. Molecular and General Genetics 124, 369-370.

Sridhara, S., Wu, T. T., Chused, T. M. \& LIN, E. C. C. (1969). Ferrous-activated nicotinamide adenine dinucleotide linked dehydrogenase from a mutant of Escherichia coli capable of growth on 1,2-propanediol. Journal of Bacteriology 98, 87-95.

TAKAGI, Y. \& SAWADA, H. (1964a). The metabolism of L-rhamnose in Escherichia coli. I. L-rhamnose isomerase. Biochemica et biophysica acta 92, 10-17.

TAKAGI, Y. \& SAWADA, H. (1964b). The metabolism of L-rhamnose in Escherichia coli. II. L-rhamnulose kinase. Biochimica et biophysica acta 92, 18-25.

WhItTAKer, R. G. \& Moss, B. A. (1981). Comparative peptide mapping at the nanomole level. Analytical Biochemistry 110, 56-60.

Ziptas, D. \& RILEY, M. (1975). Proposal concerning mechanism of evolution of the genome of Escherichia coli. Proceedings of the National Academy of Sciences of the United States of America 72, 1354-1358. 